\title{
EXPORTACIÓN Y EFICIENCIA DEL USO DE FÓSFORO EN PLÁTANO 'BARRAGANETE' (Musa paradisiaca L.)
}

\author{
EXPORTATION AND PHOSPHORUS USE EFFICIENCY \\ IN 'BARRAGANETE' BANANA (Musa paradisiaca L.)
}

\section{Leonardo Avellán-Vásquez ${ }^{1 *}$, Nexar Cobeña-Loor ${ }^{1}$, Sandra Estévez-Chica², Pablo Zamora-Macías ${ }^{3}$, Jorge Vivas-Cedeño ${ }^{1}$, Ignacio González-Ramírez ${ }^{1}$ y Adriana B. Sánchez-Urdaneta ${ }^{4}$}

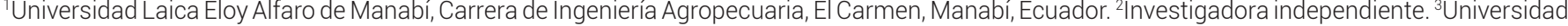
Laica Eloy Alfaro de Manabí, Carrera de Ingeniería Agropecuaria, Pedernales, Ecuador. ${ }^{4}$ Universidad del Zulia, Facultad de Agronomía, Departamento de Botánica, Maracaibo, Venezuela y Universidad Técnica de Manabí, Carrera de Ingeniería Agronómica, Manabí, Ecuador.
\end{abstract}

Autor de correspondencia: leoavellan@hotmail.com

\section{RESUMEN}

El plátano (Musa paradisiaca L.) representa el principal producto de exportación del Ecuador, especialmente de la provincia de Manabí; sin embargo, se presenta un fenómeno de deterioro de la fertilidad y degradación del suelo, por lo que existe la necesidad de mitigarlo mediante estrategias de fertilización. Se realizó un estudio en la granja experimental Río Suma, Universidad Laica Eloy Alfaro de Manabí, con el objetivo de evaluar la eficiencia de la dosis de fertilización y el fraccionamiento de $\mathrm{P}$ en plátano 'Barraganete' en una finca de Manabí, Ecuador. Se utilizó una densidad de población alta (2200 plantas ha-1) y se aplicó una dosis estándar de $100 \mathrm{~kg}$ $\mathrm{ha}^{-1}$ de N, $200 \mathrm{~kg} \mathrm{ha}^{-1}$ de $\mathrm{K}_{2} \mathrm{O}$ y $70 \mathrm{~kg} \mathrm{ha}^{-1}$ de $\mathrm{MgO}$. Los factores de estudio fueron fertilización con $P$ en tres niveles $\left(20,40\right.$ y $60 \mathrm{~kg} \mathrm{ha}^{-1}$ de $\left.\mathrm{P}_{2} \mathrm{O}_{5}\right)$ y fraccionamiento en dos niveles (100 y 40-60\% de $\left.\mathrm{P}_{2} \mathrm{O}_{5}\right)$, lo que resultó en un arreglo de tratamientos factorial $3 \times 2+1$ de tratamientos, distribuidos en un diseño experimental en bloques completos al azar con tres repeticiones; la unidad experimental estuvo conformada por 21 plantas, de las cuales se seleccionaron cinco de la parte central para las evaluaciones. Las variables evaluadas fueron eficiencia agronómica (EA), factor parcial de productividad (FPP), balance parcial de nutrientes (BPN), biomasa seca del fruto, rendimiento, concentración y exportación de $\mathrm{P}_{2} \mathrm{O}_{5}$; esta última fue mayor con $40 \mathrm{~kg} \mathrm{ha}^{-1} \mathrm{y}$ fraccionamiento de $100 \%$ (6.01 $\left.\mathrm{kg} \mathrm{ha}^{-1}\right)$. EA, FPP y BPN presentaron los valores mayores: $211.3,1002.5$ y $0.21 \mathrm{~kg} \mathrm{ha}^{-1}$ con la dosis de $20 \mathrm{~kg} \mathrm{ha}^{-1} \mathrm{y}$ fraccionamiento $40-60 \%$. El fraccionamiento $40-60$ $\%$ con dosis de $20 \mathrm{~kg} \mathrm{ha}^{-1}$ generó el rendimiento mayor $\left(20,050 \mathrm{~kg} \mathrm{ha}^{-1}\right)$. La relación entre la dosis y el fraccionamiento fue inversamente proporcional, mientras que entre el rendimiento y la eficiencia agronómica del $\mathrm{P}$ la relación fue cuadrática. Así, las recomendaciones apropiadas pueden establecerse para la fertilización del cultivo con $\mathrm{P}_{2} \mathrm{O}_{5}$

Palabras clave: Musa paradisiaca, eficiencia agronómica, extracción de nutrientes, plátano 'Barraganete'.

\section{SUMMARY}

Plantain (Musa paradisiaca L.) represents the main export product of Ecuador, especially in the province of Manabi; however, there is a phenomenon of deterioration of fertility and soil degradation, thus, there is a need to mitigate it through fertilization strategies. A study was carried out at the Río Suma experimental farm of Laic Eloy Alfaro University of Manabi, aimed to evaluate the efficiency of the fertilization dose and the fractionation of $P$ on Barraganete plantain in a farm in Manabi, Ecuador. A high population density (2200 plant ha-1) was used and a standard dose of $100 \mathrm{~kg} \mathrm{ha}^{-1}$ of $\mathrm{N}, 200 \mathrm{~kg}$ $\mathrm{ha}^{-1}$ of $\mathrm{K}_{2} \mathrm{O}$ and $70 \mathrm{~kg} \mathrm{ha}^{-1}$ of $\mathrm{MgO}$ was applied. The factors under study were $\mathrm{P}$ fertilization with three levels $\left(20,40\right.$ and $60 \mathrm{~kg} \mathrm{ha}^{-1}$ of $\left.\mathrm{P}_{2} \mathrm{O}_{5}\right)$ and fractionation with two levels (100 and $40-60 \%$ of $\mathrm{P}_{2} \mathrm{O}_{5}$ ), resulting in a $3 \times 2+1$ factorial arrangement of treatments, distributed under a randomized complete blocks experimental design with three replications; the experimental unit consisted of 21 plants, of which five were selected form the central part for the evaluations. The evaluated traits were agronomic efficiency $(\mathrm{AE})$, partial productivity factor (PPF), partial nutrient balance (PNB), dry fruit biomass, yield, concentration and exportation of $\mathrm{P}_{2} \mathrm{O}_{5}$; the latter was higher with the dose of $40 \mathrm{~kg} \mathrm{ha}^{-1}$ and fractionation of $100 \%\left(6.01 \mathrm{~kg} \mathrm{ha}^{-1}\right) ;$ AE, PPF and PNB presented the highest values: $211.3,1,002.5$ and $0.21 \mathrm{k} \mathrm{ha}^{-1}$, respectively with the dose of $20 \mathrm{~kg} \mathrm{ha}^{-1}$ and the fractionation $40-60 \%$. The fractionation $40-60 \%$ with a dose of 20 $\mathrm{kg} \mathrm{ha}^{-1}$ produced the highest yield $\left(20,050 \mathrm{~kg} \mathrm{ha}^{-1}\right)$. The relationship between dose and fractionation was inversely proportional, while between yield and agronomic efficiency of $\mathrm{P}$ the relationship was quadratic. Thus, appropriate recommendations can be established for fertilizing the crop with $\mathrm{P}_{2} \mathrm{O}_{5}$.

Index words: Musa paradisiaca, agronomic efficiency, 'Barraganete' plantain, nutrient extraction.

\section{INTRODUCCIÓN}

La mayor producción de plátano (Musa paradisiaca) corresponde a África con $72.7 \%$, América (22.9 \%) y Asia (4.3\%) (FAOSTAT, 2018). De acuerdo con Sepúlveda et al. (2017), los mayores productores en el continente americano son Colombia (38.1 \%), Perú (21.6\%), República Dominicana (6.9 \%) y Ecuador (6.4\%). En cuanto al comercio, para el año 2014 se exportó plátano globalmente por un valor de 470.2 millones de dólares; para ese mismo año, la producción mundial de plátano se incrementó en 1.93 \% con respecto al año 2012, y alcanzó la cifra más alta en el periodo comprendido entre los años 2000 y 2014 (MAGAP, 2014). En Ecuador, el cultivo de plátano, además de ser un producto tradicional, genera importantes divisas y ocupó el primer lugar en las exportaciones mundiales con 97.3 millones de dólares (Sepúlveda et al., 2017). De acuerdo con el Instituto Nacional de Estadística y Censos (INEC, 2018), Ecuador se encuentra entre los 10 países con mayor producción de plátano, con un estimado de 
141,441 ha y 604,134 t de producción (FAOSTAT, 2018). La provincia de Manabí, ubicada al suroeste del país, es la mayor productora con 50,376 ha; de ellas, el cantón El Carmen, ubicado al norte de la provincia, representa el $35.62 \%$ de la superficie nacional sembrada (INEC, 2014). A nivel nacional, el $71.6 \%$ del cultivo de plátano se ha establecido como monocultivo, lo que indica cierto grado de especialización de la producción (Sepúlveda et al., 2017).

Existe la necesidad de revertir el deterioro de la fertilidad del suelo y la degradación de la base de los recursos naturales (tierra, agua, bosque y biodiversidad) para aumentar y mantener la productividad de los cultivos, lo que a su vez incrementaría la seguridad y soberanía alimentaria y reduciría la falta de alimentos y la pobreza (Adamtey et al., 2016). Las opciones recomendadas con más frecuencia para lograrlo son desarrollar la agricultura convencional, diversificar los sistemas de producción o intensificar los patrones de producción existentes (Dixon et al., 2001; Folberth et al., 2014); en este sentido, uno de los propósitos del uso de los nutrientes es aumentar el rendimiento general de los cultivos al suministrar la fuente de sustento requerido, a la vez que se disminuyen las pérdidas de estos nutrientes de los suelos dedicados a la producción (Fixen et al., 2015).

Factores como el tipo de suelo y el cultivo influyen en el balance de $\mathrm{N}$ y P, así como factores de manejo (tipo y cantidad de fertilizante o estiércol) y las propiedades del suelo (estructura, profundidad del enraizamiento y contenido de materia orgánica), tal como lo señalaron Lipiec y Stępniewski (1995), Oenema et al. (2010) y van Leeuwen et al. (2019).

Para incrementar la producción, los agricultores usan mezclas de diferentes fertilizantes en cantidades grandes; sin embargo, pocas veces se tiene en cuenta la eficiencia del uso de los nutrientes (EUN), definida como la biomasa total producida por la planta por unidad de nutrientes absorbidos (Gourley et al., 1994). Las investigaciones sobre la EUN no son recientes, tienen diferentes aplicaciones y reflejan la preocupación por el uso eficiente que las plantas pueden hacer de los nutrientes (Doyle y Holford, 1993), pero es evidente que en la actualidad cobra interés por el costo de los fertilizantes y el impacto ambiental que éstos generan (Fageria et al., 2010; Fallah y Tadayyon, 2010; Weih et al., 2010).

El $\mathrm{P}$ es un elemento indispensable en la agricultura, ya que no puede ser sustituido por ningún otro, ni obtenido de la atmósfera para la producción de los cultivos. Se requiere un suministro adecuado de $\mathrm{P}$ para un crecimiento y reproducción óptimos. La concentración total de P en los cultivos agrícolas generalmente varía de 0.1 a $0.5 \%$. Una vez en el interior de las raíces, el $P$ puede almacenarse en la raíz o transportarse a las partes superiores de la planta. A través de diversas reacciones químicas se incorpora a compuestos orgánicos, incluidos los ácidos nucleicos (ADN y ARN), fosfoproteínas, fosfolípidos, fosfatos de azúcar, enzimas y compuestos de fosfato ricos en energía como el trifosfato de adenosina (ATP). Es en estas formas orgánicas, así como ion fosfato inorgánico, que el $\mathrm{P}$ se mueve por toda la planta donde está disponible para otras reacciones (Bhattacharya, 2018).

Una fertilización eficiente debe tener presente los factores que la afectan, entre ellos la exportación de nutrientes (EN), que se refiere a la cantidad de nutrientes que se extraen de la plantación y se transfieren al fruto (Cruzate y Casas, 2003); la eficiencia agronómica (EA) se refiere al incremento de los rendimientos por unidad de nutrientes (Sánchez et al., 2012); el factor parcial de productividad (FPP) corresponde al rendimiento del cultivo por unidad de nutriente aplicado (Bruulsema et al., 2008) y el balance parcial de nutrientes (BPN) está dado por la diferencia entre la cantidad de nutriente que ingresa y la que se extrae de la plantación (Ciampitti y García, 2008). Por las razones expuestas, el objetivo del presente estudio fue evaluar la eficiencia de la dosis de fertilización y el fraccionamiento de $\mathrm{P}$ en plátano 'Barraganete' en la provincia de Manabí, Ecuador.

\section{MATERIALES Y MÉTODOS}

\section{Sitio experimental}

El estudio se realizó en la Granja Experimental Río Suma de la Universidad Laica Eloy Alfaro de Manabí, ubicada en el cantón El Carmen, provincia de Manabí, Ecuador $\left(0^{\circ} 15^{\prime}\right.$ S, $79^{\circ} 26^{\prime}$ O, 260 msnm de altitud); el clima es de trópico húmedo, temperatura media anual de $24{ }^{\circ} \mathrm{C}$, precipitación anual de $2806 \mathrm{~mm}, 86 \%$ de humedad relativa y 1026.2 horas luz año-1 (INAMHI, 2017).

El análisis de suelo del lote experimental presentó una densidad aparente de $0.93 \mathrm{~g} \mathrm{~cm}^{-3}$, un contenido de materia orgánica (MO) medio (4.48\%), conductividad eléctrica (CE) de $0.08 \mathrm{ds} \mathrm{m}^{-1}, \mathrm{pH}$ medianamente ácido (5.77). Niveles bajos de $\mathrm{NH}_{4}$ (11.61 ppm), P (4.56 ppm), S (2.14 ppm), Mg $\left(0.90\right.$ meq $\left.100 \mathrm{~g}^{-1}\right)$, sumatoria de bases de 8.40 meq 100 $\mathrm{g}^{-1}, \mathrm{Mn}(9.70 \mathrm{ppm})$, relación Mg/K (1.80), Ca+Mg/K (15.80), niveles altos de $\mathrm{K}\left(0.50 \mathrm{meq} 100 \mathrm{~g}^{-1}\right)$, Ca $\left(7.00 \mathrm{meq} 100 \mathrm{~g}^{-1}\right)$, Cu (5.80 ppm), Fe (123.1 ppm), Zn (23.20 ppm) y relación $\mathrm{Ca} / \mathrm{Mg}$ (7.78). La textura es franco arenosa (62\% de arena, $20 \%$ de limo y $10 \%$ de arcilla). 


\section{Manejo agronómico}

Se utilizaron plantas de plátano 'Barraganete' en densidad alta (2200 plantas ha-1), las cuales fueron trasplantadas al emitir la hoja cinco; posteriormente, se aplicó una dosis estándar de fertilización de $100 \mathrm{~kg} \mathrm{ha}^{-1}$ de N, $200 \mathrm{~kg} \mathrm{ha}^{-1}$ de $\mathrm{K}_{2} \mathrm{O}$ y $70 \mathrm{~kg} \mathrm{ha}^{-1}$ de $\mathrm{MgO}$, fraccionados en tres partes iguales y aplicado al suelo cuando la planta emitió las hojas seis, 12 y 18.

\section{Arreglo de tratamientos y diseño experimental}

Los tratamientos consistieron en la combinación de dos factores: 1) dosis de fertilización con tres niveles (20, 40 y $60 \mathrm{~kg} \mathrm{ha}^{-1}$ de $\mathrm{P}_{2} \mathrm{O}_{5}$ ) y 2) fraccionamiento del fertilizante con dos niveles ( $100 \%$ y $40-60 \%$ de $\mathrm{P}_{2} \mathrm{O}_{5}$ ), dando como resultado un arreglo factorial $3 \times 2+1$. Los tratamientos se establecieron en un diseño experimental de bloques completos al azar con tres repeticiones; la unidad experimental estuvo conformada por 21 plantas, de las cuales se seleccionaron cinco de la parte central para evitar el efecto de bordo y sobre ellas se realizaron las evaluaciones.

\section{Variables evaluadas}

\section{Exportación de P (EXP)}

Se determinó en laboratorio mediante la cantidad del macronutriente $\left(\mathrm{P}_{2} \mathrm{O}_{5}\right)$ extraído por el fruto, expresado en $\mathrm{kg} \mathrm{ha}^{-1}$, sin incluir el raquis. El proceso consistió en la toma de $1 \mathrm{~kg}$ de los frutos con calidad de exportación como muestra, provenientes de los dos dedos centrales de la segunda mano, seguido de la toma de su biomasa fresca, y 3 d después de que las muestras fueron colocadas en estufa universal UN30 (Memmert GmbH, Buchembach, Alemania) a temperatura de $65^{\circ} \mathrm{C}$ se obtuvo su biomasa seca constante; posteriormente, se unificaron las muestras de las plantas correspondientes a la misma unidad experimental para ser molidas y preparadas para su análisis en el laboratorio de suelos, tejidos y aguas de la Estación Experimental Tropical Pichilingue del Instituto Nacional de Investigaciones Agropecuarias de Ecuador, donde se obtuvo el porcentaje de biomasa seca, que debió ser transformado en biomasa fresca, y tras considerar el rendimiento del cultivo en $\mathrm{kg} \mathrm{ha}^{-1}$ se calculó la cantidad de nutriente exportado (Cruzate y Casas, 2003).

\section{Eficiencia agronómica (EA)}

Para su estimación se emplearon los rendimientos de las parcelas y las dosis evaluadas mediante la fórmula:

$$
E A=\left(R_{N}-R_{0}\right) / D
$$

donde: $R_{N}$ es el rendimiento de la parcela fertilizada $\left(\mathrm{kg} \mathrm{ha}^{-1}\right)$, $\mathrm{R}_{0}$ es el rendimiento de la parcela sin fertilizar $\left(\mathrm{kg} \mathrm{ha}^{-1}\right)$ y $\mathrm{D}$ es la dosis de nutriente $\left(\mathrm{kg} \mathrm{ha}^{-1}\right)$.

\section{Factor parcial de productividad (FPP)}

Se utilizó el procedimiento de Bruulsema et al. (2008), como se indica en la siguiente ecuación:

$$
F P P=R_{N} / D
$$

donde: $R_{N}$ es el rendimiento del cultivo $\left(\mathrm{kg} \mathrm{ha}^{-1}\right)$ y $\mathrm{D}$ es la dosis de nutriente $\left(\mathrm{kg} \mathrm{ha}^{-1}\right)$.

\section{Balance parcial de nutrientes (BPN)}

Se estimó mediante el procedimiento de Ciampitti y García (2008) utilizando la fórmula siguiente:

$$
B N P=C_{N} / D_{N}
$$

donde: $\mathrm{C}_{\mathrm{N}}$ es la exportación de nutrientes en el fruto $(\mathrm{kg}$ $\left.\mathrm{ha}^{-1}\right)$ y DN es la dosis de nutriente $\left(\mathrm{kg} \mathrm{ha}^{-1}\right)$.

\section{Análisis estadístico}

A cada variable se le aplicó análisis de varianza y los promedios de los tratamientos se compararon mediante la prueba de Tukey $(\mathrm{P} \leq 0.05)$; adicionalmente, se aplicaron análisis de regresión y se calcularon líneas de tendencia para visualizar el comportamiento de la exportación y eficiencia con base en los resultados obtenidos a través de las diferentes dosis y fraccionamientos de P aplicados.

\section{RESULTADOS Y DISCUSIÓN}

\section{Exportación de fósforo}

La cantidad mayor de $\mathrm{P}$ exportado en el fruto se presentó con la dosis de $40 \mathrm{~kg} \mathrm{ha}^{-1} \mathrm{y}$ la fracción de 100 $\%$, la cual fue de $6.01 \mathrm{~kg} \mathrm{ha}^{-1}$; la dosis de $60 \mathrm{~kg} \mathrm{ha}^{-1}$ en la fracción 40-60 \% generó la exportación menor (3.11 $\left.\mathrm{kg} \mathrm{ha}^{-1}\right)$, con diferencias estadísticas $(P<0.001)$ entre estos dos tratamientos (Cuadro 1). Todas las dosis y fraccionamientos fueron diferentes estadísticamente entre sí $(P<0.0031)$, con excepción de las dosis de 20 y $40 \mathrm{~kg} \mathrm{ha}^{-1}$ con fraccionamiento de la aplicación de 40-60\%, que fueron estadísticamente similares ( $P$ > 0.257 ) con exportación de 4.28 y $4.22 \mathrm{~kg} \mathrm{ha}^{-1}$. En este sentido, Avellán et al. (2015) señalaron que aun cuando el $\mathrm{P}$ cumple diversas funciones en la planta, la cantidad absorbida y requerida por el cultivo es muy baja, indicaron además que en plátano 'Barraganete' cultivado en la Finca Santa Marianita en El Carmen, Manabí, con una densidad 
de 2,500 plantas ha-1, el total absorbido fue de $32.88 \mathrm{~kg} \mathrm{ha}^{-1}$ y el exportado en los dedos y el raquis fue de $7.72 \mathrm{~kg} \mathrm{ha}^{-}$ ${ }^{1}$, contenido superior al encontrado en esta investigación. Dichos autores resaltaron que los productores generalmente no fertilizan con este elemento, ya que se encuentra en los suelos de la zona en contenidos de entre 10 y 18 ppm (20 a $36 \mathrm{~kg} \mathrm{ha}^{-1}$ ) de P total o disponible

El P es un elemento de baja movilidad en el suelo, por lo que las raíces pueden absorber el que se encuentra alrededor de ellas. Al añadir al suelo los fertilizantes, el $P$ se encuentra en forma soluble y disponible, pero éste rápidamente es fijado y deja de estar disponible para la planta. Dada esa baja movilidad, el P permanece en la capa superior del suelo y las pérdidas ocurren principalmente a través de escorrentía superficial y erosión; por ello, el fraccionamiento del $\mathrm{P}$ es considerado con el fin de incrementar su eficiencia. Los suelos provenientes de cenizas volcánicas (Andisoles), por su composición de arcillas, tienen la característica de fijar el P, por lo que su aplicación es ineficiente en estos suelos; por tal motivo, el fraccionamiento permitiría que la planta asimile un mayor contenido del nutriente, ya que al ser aplicado en una sola dosis, lo que la planta no logra absorber es fijado en el suelo; sin embargo, el fraccionarlo permitiría tener dos momentos de absorción y éste sería mejor utilizado por la planta.

La cantidad de nutrientes extraídos por una planta durante su ciclo de vida se podría determinar a través de la elaboración de curvas de extracción de nutrientes, así se conocerían las épocas de mayor absorción y extracción de cada nutriente, permitiría establecer programas de fertilización adecuados para cada cultivo (Avellán et al.,
2015; Castillo et al., 2011) y considerar tanto la cantidad de fertilizante como la fase fenológica apropiada para realizar las aplicaciones (Bertsch, 2003).

De acuerdo con Castillo et al. (2011), el ciclo de vida del plátano consta de tres etapas: vegetativa, floración y fructificación. El proceso de absorción, reciclaje y reposición de los nutrientes es muy dinámico, alcanza su punto máximo en las etapas de floración y llenado de los frutos. Los mismos autores estimaron que la extracción total de P en plátano 'Dominico' fue de $29 \mathrm{~kg} \mathrm{ha}^{-1}$ con una densidad de 1875 plantas ha-1. El racimo de esa cantidad de unidades por hectárea extrajo $3.2 \mathrm{~kg} \mathrm{ha}^{-1} \mathrm{de}$ P y alcanzó una biomasa fresca promedio de $9 \mathrm{~kg}$; una tonelada de fruta extrajo aproximadamente $189.6 \mathrm{~g}$ de P. De acuerdo con Dobermann (2005), los nutrientes que no son absorbidos por el cultivo en un ciclo de crecimiento no necesariamente se pierden del sistema, sino que son utilizados por los futuros cultivos; esta aseveración coincide con lo señalado por Castillo et al. (2011), quienes indicaron que las musáceas acumulan el $\mathrm{P}$ que requieren por un largo periodo, pierden relativamente poco a través del fruto y lo redistribuyen fácilmente a la planta hija.

De acuerdo con Vivas-Cedeño et al. (2018) la fertilización con $\mathrm{P}$ no tuvo efecto en el rendimiento del cultivo de plátano 'Barraganete' en las localidades de Sumita Pita, Las Palmitas de Agua Sucia y La Y de la Raíz (Cantón El Carmen, Manabí); dichos resultados se contraponen con los obtenidos en esta investigación, donde con niveles bajos de P inicial (4.56 ppm) se encontraron diferencias en el rendimiento del cultivo que estuvieron entre 20,050 y $18,150 \mathrm{~kg} \mathrm{ha}^{-1}$ por la aplicación de diferentes dosis y fraccionamiento de $\mathrm{P}$,

Cuadro 1. Rendimiento, biomasa seca, concentración de $\mathrm{P}$, exportación, eficiencia agronómica, factor parcial de productividad y balance parcial de nutrientes de $\mathrm{P}_{2} \mathrm{O}_{5}$ en el fruto de plátano 'Barraganete'.

\begin{tabular}{lcccccccc}
\hline Dosis $\left(\mathrm{kg} \mathrm{ha}^{-1}\right)$ & Fracción (\%) & $\begin{array}{c}\text { Rendimiento } \\
\left(\mathrm{kg} \mathrm{ha}^{-1}\right)\end{array}$ & BS $\left(\mathrm{kg} \mathrm{ha}^{-1}\right)$ & \begin{tabular}{l}
{$[\mathrm{P}](\%)$} \\
\hline 0
\end{tabular} & $\begin{array}{c}\text { Exportación de } \\
\mathrm{P}\left(\mathrm{kg} \mathrm{ha}^{-1}\right)\end{array}$ & EA & FPP & BPN \\
\hline 20 & $40-60$ & $20,050 \mathrm{a}$ & $6116.31 \mathrm{a}$ & $0.07 \mathrm{a}$ & $4.28 \mathrm{c}$ & $211.3 \mathrm{a}$ & $1002.5 \mathrm{a}$ & $0.21 \mathrm{a}$ \\
40 & $40-60$ & $18,775 \mathrm{a}$ & $6027.47 \mathrm{a}$ & $0.07 \mathrm{a}$ & $4.22 \mathrm{c}$ & $73.8 \mathrm{c}$ & $69.4 \mathrm{e}$ & $0.11 \mathrm{~d}$ \\
60 & $40-60$ & $13,925 \mathrm{~b}$ & $3885.44 \mathrm{~b}$ & $0.08 \mathrm{a}$ & $3.11 \mathrm{e}$ & $-31.7 \mathrm{~d}$ & $232.1 \mathrm{~d}$ & $0.05 \mathrm{f}$ \\
20 & 100 & $18,150 \mathrm{a}$ & $4446.64 \mathrm{~b}$ & $0.08 \mathrm{a}$ & $3.56 \mathrm{~d}$ & $116.3 \mathrm{~b}$ & $907.5 \mathrm{a}$ & $0.18 \mathrm{~b}$ \\
40 & 100 & $19,650 \mathrm{a}$ & $6678.60 \mathrm{a}$ & $0.09 \mathrm{a}$ & $6.01 \mathrm{a}$ & $95.6 \mathrm{~b}$ & $491.3 \mathrm{~b}$ & $0.15 \mathrm{c}$ \\
60 & 100 & $19,975 \mathrm{a}$ & $6246.39 \mathrm{a}$ & $0.08 \mathrm{a}$ & $5.00 \mathrm{~b}$ & $69.2 \mathrm{c}$ & $332.9 \mathrm{c}$ & $0.08 \mathrm{e}$ \\
$\mathrm{DSH}_{0.05}$ & & 2187.23 & 1297.76 & 0.03 & 0.1918 & 21.47 & 98.71 & 0.0117 \\
\hline
\end{tabular}

Medias con letras iguales en las columnas no son estadísticamente diferentes (Tukey, 0.05). BS: biomasa seca, [P]: concentración de P (\%), EA: eficiencia agronómica, FPP. factor parcial de productividad, BPN: balance parcial de nutrientes, DSH: diferencia significativa honesta. 
con excepción de la dosis de $60 \mathrm{~kg} \mathrm{ha}^{-1}$ y fraccionamiento de $40-60 \%$, que obtuvo $13,925 \mathrm{~kg} \mathrm{ha}^{-1}$, que fue el menor rendimiento obtenido en esta investigación.

\section{Eficiencia agronómica (EA)}

La eficiencia del uso de los nutrientes es la forma idónea de evaluar la respuesta del cultivo de plátano al uso de fertilizantes. La eficiencia de los nutrientes consiste en obtener el máximo aprovechamiento de la fertilización para incrementar el rendimiento de los cultivos por unidad de nutriente aplicada (Stewart, 2007). De acuerdo con Espinosa y García (2009), la manera más eficiente de evaluar esta variable es a través de la eficiencia agronómica, la cual se calcula mediante la diferencia del rendimiento de la parcela fertilizada y aquella sin fertilizar.

Se encontraron diferencias $(P<0.0001)$ en la EA por efecto de los tratamientos. Con la dosis de $20 \mathrm{~kg} \mathrm{ha}^{-1} \mathrm{y}$ fraccionada 40-60 \% se obtuvo una EA de $211.3 \mathrm{~kg} \mathrm{ha}^{-1}$ en comparación con la dosis de $60 \mathrm{~kg} \mathrm{ha}^{-1}$ con fraccionamiento 40-60\%, que presentó una EA negativa de $-31.7 \mathrm{~kg} \mathrm{ha}^{-1}$ (Cuadro 1, Figuras $1 \mathrm{~A}$ y $\mathrm{B})$, ambas resultaron estadísticamente diferentes entre sí $(P>0.003)$. En la fracción $100 \%$ con dosis de 20 y $40 \mathrm{~kg} \mathrm{ha}^{-1}$ se obtuvo una EA de 116.32 y 95.6 $\mathrm{kg} \mathrm{ha}^{-1}$ (Cuadro 1), sin diferencias estadísticas entre ambos $(P>0.4671)$; igualmente, con las dosis de 40 y $60 \mathrm{~kg}$ ha${ }^{1}$ con fraccionamiento de $40-60$ y $100 \%$, respectivamente, no se presentaron diferencias estadísticas $(P>0.0856)$ entre ambos tratamientos (Cuadro 1). Se observó que con ambos fraccionamientos (40-60 y $100 \%$ ) las mayores EA se obtuvieron con la aplicación de $20 \mathrm{~kg} \mathrm{ha}^{-1}$ de $\mathrm{P}_{2} \mathrm{O}_{5}$ (211.3 y $116.3 \mathrm{~kg} \mathrm{ha}{ }^{-1}$, respectivamente), diferentes estadísticamente entre ellas $(P<0.0023)$. Las dosis de 40 y $60 \mathrm{~kg} \mathrm{ha}^{-1}$ siguen ese mismo patrón de EA, la cual fue intermedia (73.8 y $69.2 \mathrm{~kg} \mathrm{ha}^{-1}$ ) para el fraccionamiento 4060 y $100 \%$, respectivamente, sin diferencias estadísticas ( $P$ $>0.079$ ) entre ellas (Cuadro 1)

Los resultados obtenidos en esta investigación fueron superiores a los obtenidos en el cultivo de plátano Barraganete con una densidad de 1700 plantas ha-1 en $^{-1}$ la localidad de Sumita Pita (norte de Manabí), donde se reportó que la EA promedio fue de $116 \mathrm{~kg} \mathrm{ha}^{-1}$ con la dosis de $60 \mathrm{~kg} \mathrm{ha}^{-1}$ de $\mathrm{P}_{2} \mathrm{O}_{5}$ (Vivas-Cedeño et al., 2018). Furcal-Beriguete y Barquero-Badilla (2013) encontraron en plátano Curare semi gigante con dosis de $70 \mathrm{~kg} \mathrm{ha}^{-1}$ de $\mathrm{P}_{2} \mathrm{O}_{5}$ extracciones de $\mathrm{P}$ de 41.79 y $67.55 \%$ en dedos comerciales y de 58.21 y $32.45 \%$ en dedos no comerciales para los años 2009 y 2010, respectivamente. De acuerdo con Combatt et al. (2004), no es conveniente considerar una dosis general de nutrimentos para ser recomendada, pues ésta depende del contenido de nutrientes presente en el suelo.
Es importante destacar que la EA negativa para la dosis de $60 \mathrm{~kg} \mathrm{ha}^{-1}$ con fraccionamiento 40-60 \% (-31.7 $\mathrm{kg} \mathrm{ha}^{-1}$ ) se debió a que el rendimiento en las parcelas no fertilizadas $\left(15,825 \mathrm{~kg} \mathrm{ha}^{-1}\right)$ fue superior que en algunas de las parcelas fertilizadas $\left(13,925 \mathrm{~kg} \mathrm{ha}^{-1}\right.$ ) (Cuadro 1, Figuras $1 \mathrm{~A}$ y 1B). Lo anterior es el resultado de que la absorción del nutriente surtió un efecto negativo en la planta, la absorción del nutriente por exceso de fertilización es superior al requerimiento de la planta, probablemente la toma de este nutriente impide la absorción de otros nutrientes requeridos por la planta en mayor volumen y se genera un desbalance; ésto es, más $P$ del requerido y menos de otros nutrientes, especialmente N; por tal motivo, disminuye el rendimiento con esa dosis elevada de P.

En concordancia con estos resultados, Vivas-Cedeño et al. (2018) encontraron EA negativa en algunas localidades y para determinadas dosis de nutrientes, y señalaron que ésto obedeció a que el rendimiento de algunas parcelas sin fertilizantes superó a las fertilizadas, por lo que concluyeron que a mayor uso de nutrientes la producción disminuyó a partir de la aplicación, lo que se atribuyó a que la respuesta del rendimiento al uso de fertilizantes depende del sitio de establecimiento del cultivo; además, Fixen (2010) señaló que el $\mathrm{P}$ y el $\mathrm{K}$ tienen una fácil acumulación en el suelo en formas disponibles para las plantas; en este orden de ideas, Stewart (2007) indicó que resulta necesario evaluar la eficiencia y recuperación de nutrientes a largo plazo.

Fixen et al. (2015) concluyeron que la eficiencia del uso de nutrientes (NUE) es un concepto de importancia crítica en la evaluación de los sistemas de producción de cultivos. El manejo de los fertilizantes, el agua del suelo y de las plantas podrían afectar su impacto en gran medida. El uso de los nutrientes tiene por objeto aumentar el rendimiento general de los sistemas de cultivo, lo que proporciona una nutrición económicamente óptima para el cultivo y minimiza las pérdidas de nutrientes del campo; además, los niveles más bajos de EA sugieren que los cambios en el manejo podrían aumentar la respuesta de los cultivos o reducir los costos de producción.

Por otro lado, aun cuando existe poca información sobre este tópico de la fertilización en plátano, las nuevas investigaciones están dirigidas a plantaciones jóvenes, sin considerar que en la mayoría de los casos los productores manejan cultivos en segundo ciclo o ya establecidos (Vivas-Cedeño et al., 2018).

\section{Factor parcial de productividad (FPP)}

Se encontraron diferencias estadísticas entre los tratamientos evaluados ( $\mathrm{P} \leq \mathrm{0.0001})$, con excepción de la dosis de $20 \mathrm{~kg} \mathrm{ha}^{-1}$ con fraccionamientos de 

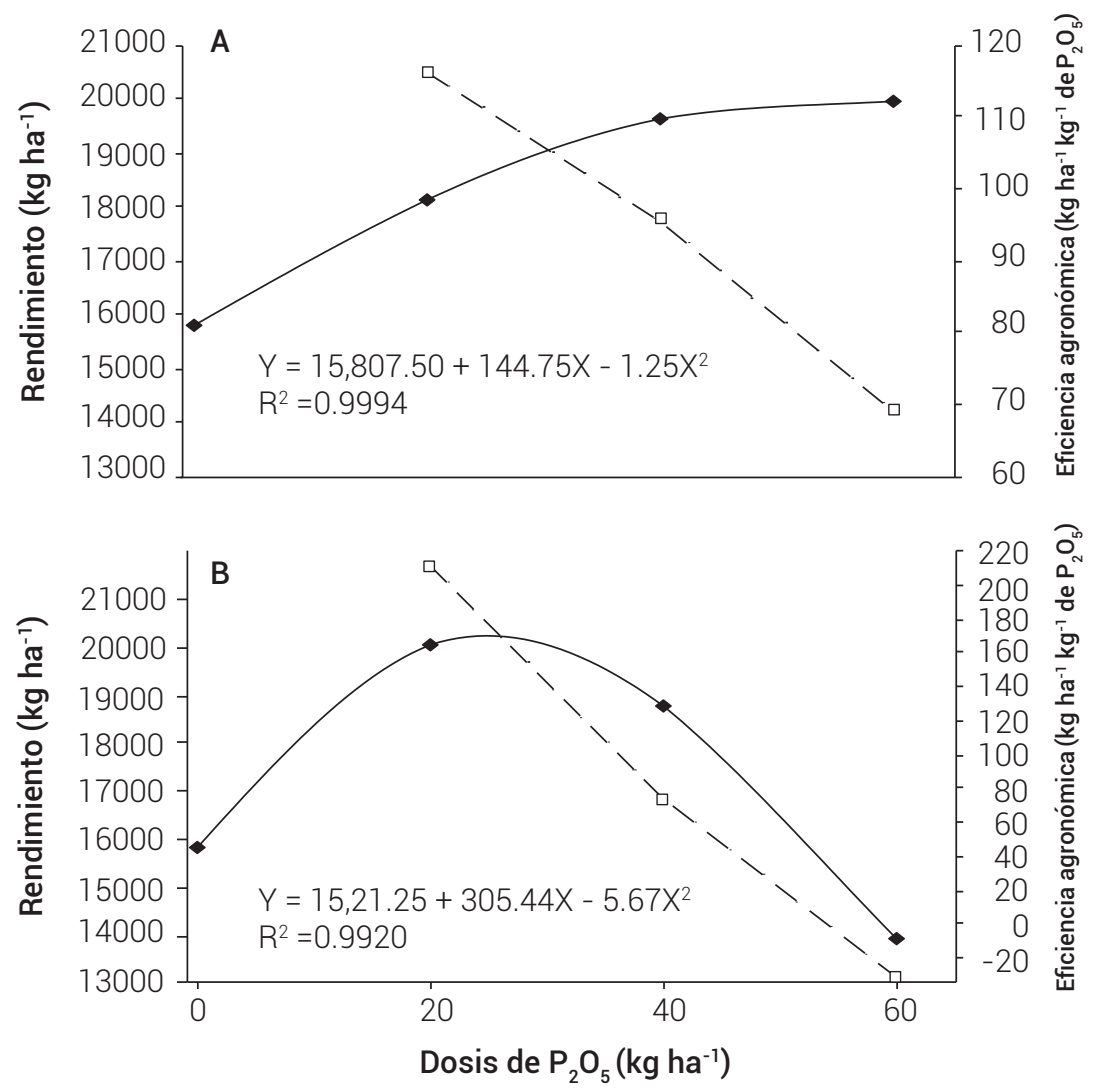

Figura 1. Eficiencia agronómica de $\mathrm{P}_{2} \mathrm{O}_{5}$ con dosis de $0,20,40$ y $\left.60 \mathrm{~kg} \mathrm{ha}^{-1}, \mathrm{~A}\right)$ con fracción del $100 \%$ y $\mathrm{B}$ ) con fracción 40-60\%. = rendimiento, $\square=$ eficiencia agronómica.

40-60 y $100 \%$, los cuales resultaron no significativos ( $P$ $>0.0954)$. Con los fraccionamientos de 100 y $40-60 \%$ y dosis de $20 \mathrm{~kg} \mathrm{ha}^{-1}$ se obtuvieron 907.5 y $1002.5 \mathrm{~kg} \mathrm{ha}^{-1}$ de rendimiento de fruto por $\mathrm{kg}$ de nutriente aplicado, sin diferencias estadísticas $(P>0.3719)$ entre ellos se acepta la sugerencia (Cuadro 1). En el FPP, con relación a la EA para los dos fraccionamientos con la dosis de $20 \mathrm{~kg} \mathrm{ha}^{-1}$ se obtuvieron los valores de eficiencia más elevados, ya que la EA se estimó comparando con el testigo, mientras el FPP estimó el rendimiento por kg de nutriente aplicado; por esta razón, la EA fue mayor y sobresalió en los valores de FPP con las dosis de 20 y $40 \mathrm{~kg} \mathrm{ha}^{-1}$ con fraccionamiento de $40-60$ y $100 \%$, respectivamente.

Estos resultados fueron superiores a los encontrados en plátano 'Barraganete' con dos dosis de fósforo (60 y $120 \mathrm{~kg} \mathrm{ha}^{-1}$ ) en un cultivo establecido, en el cual se obtuvo un FPP promedio de 257.66 y 125.67 , respectivamente (Vivas-Cedeño et al., 2018). Es importante destacar que los resultados obtenidos en esta investigación para FPP fueron superiores 3.89 y 7.22 veces, respectivamente, al compararlos con 1002.5 y $907.5 \mathrm{~kg} \mathrm{ha}^{-1}$, generados en un cultivo renovado.
El FPP mostró valores superiores a los obtenidos en plátano 'Curare' con dosis de $70 \mathrm{~kg} \mathrm{ha}^{-1}$ de $\mathrm{P}_{2} \mathrm{O}_{5}$ donde obtuvieron en promedio $315 \mathrm{~kg}$ de fruto por $\mathrm{kg}$ de nutriente (Furcal-Beriguete y Barquero-Badilla, 2013). Boaretto et al. (2007) señalaron que a mayor aplicación del fertilizante la eficiencia productiva de los cultivos menor fue; es decir, que la eficiencia del nutriente fue alta con dosis bajas de fertilización.

Fixen et al. (2015) encontraron niveles menores de FPP en suelos menos sensibles a la sobre aplicación de nutrientes, los niveles mayores indicaron que el suministro de nutrientes probablemente limitó la productividad. De acuerdo con estos resultados, la aplicación óptima de $\mathrm{P}_{2} \mathrm{O}_{5}$ estaría entre 30 y $40 \mathrm{~kg} \mathrm{ha}^{-1}$, con rendimientos cercanos a los 19,000 kg ha-1 (Figura 2).

\section{Balance parcial de nutriente (BPN)}

Se encontraron diferencias significativas $(P<0.0001)$ para BPN por efecto de los tratamientos; éste fue mayor con $20 \mathrm{~kg} \mathrm{ha}^{-1} \mathrm{y}$ fue disminuyendo conforme las dosis aumentaron en ambos fraccionamientos (Figura 3). Tanto en el fraccionamiento de 100 como el de $40-60 \%$, el BPN 


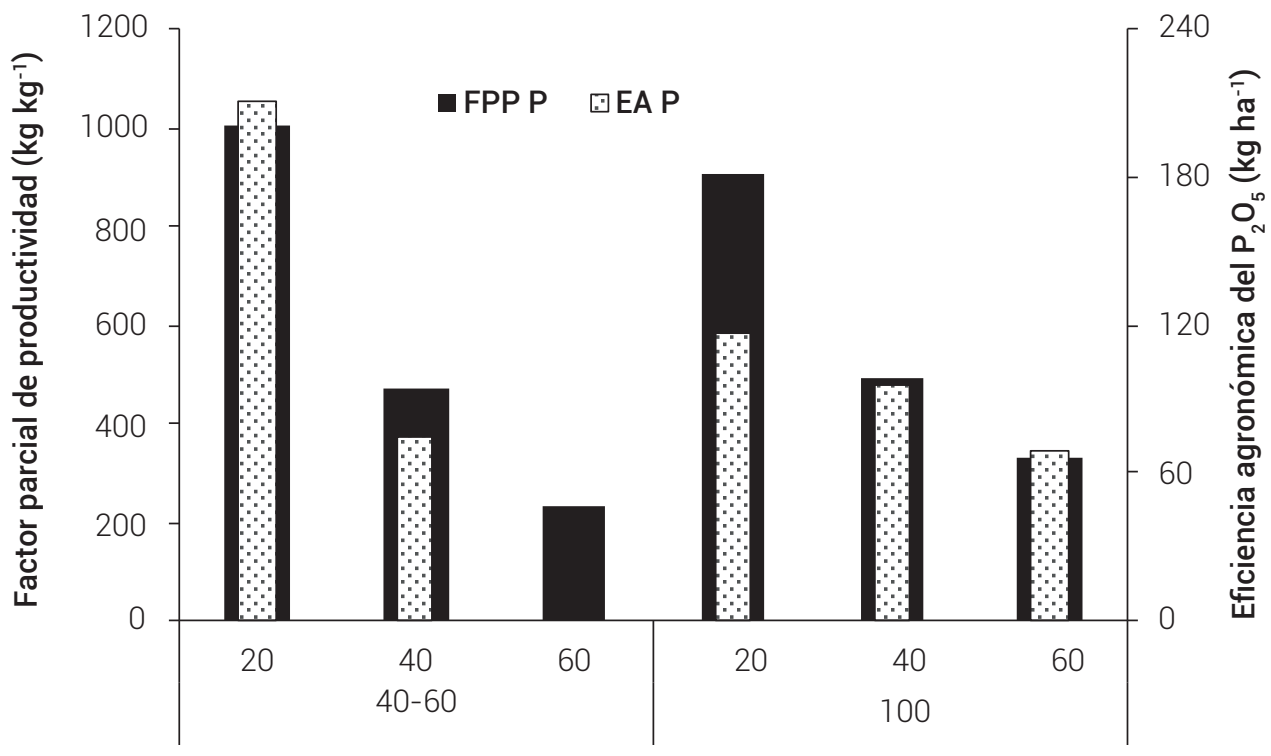

Dosis de $\mathrm{P}_{2} \mathrm{O}_{5}\left(\mathrm{~kg} \mathrm{ha}^{-1}\right)$ y fraccionamientos (\%)

Figura 2. Factor parcial de productividad de $\mathrm{P}_{2} \mathrm{O}_{5}$ en fracciones 100 y 40-60\%. FPP P. factor parcial de productividad de fósforo y EA P. eficiencia agronómica del fósforo.

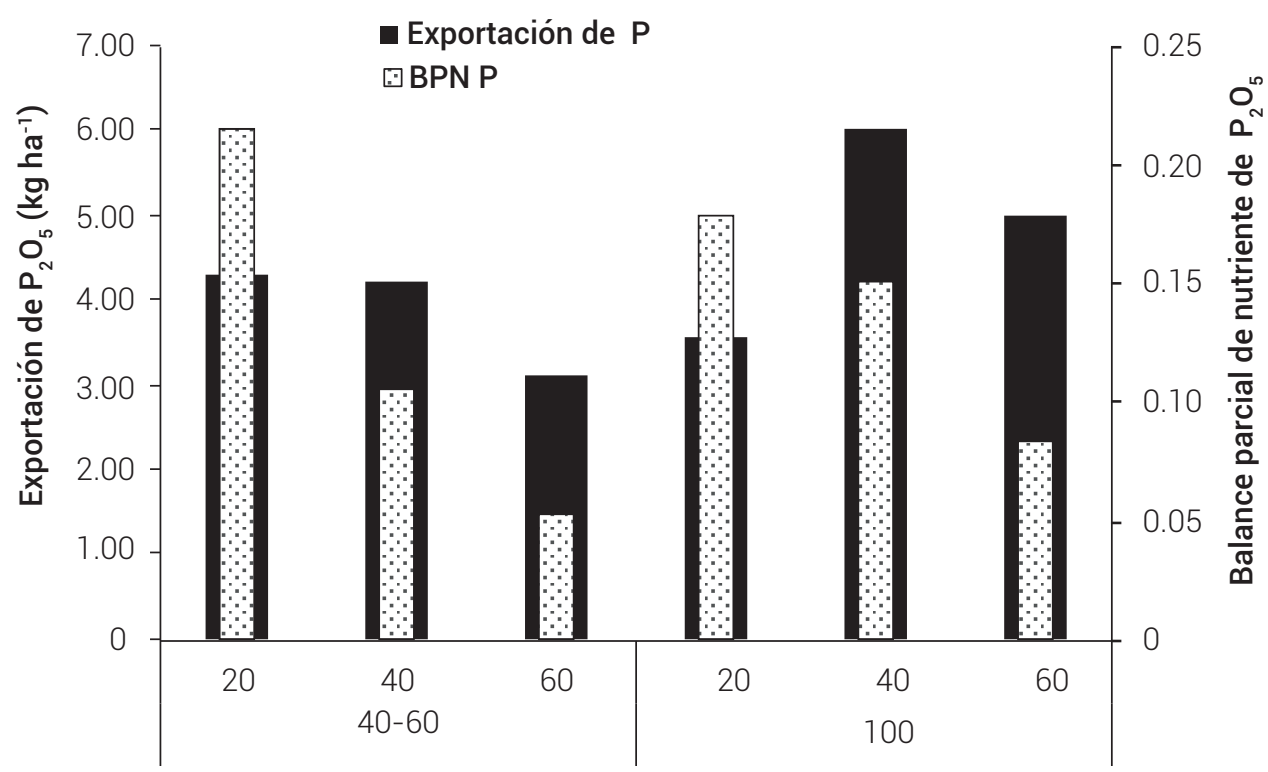

Dosis de $\mathrm{P}_{2} \mathrm{O}_{5}\left(\mathrm{~kg} \mathrm{ha}^{-1}\right)$ y fraccionamientos (\%)

Figura 3. Balance parcial de nutrientes de $\mathrm{P}_{2} \mathrm{O}_{5}$ en fracciones 100 y $40-60 \%$. P. fósforo y BPN P. balance parcial de nutrientes de fósforo.

fue mayor que la exportación de nutrientes con la dosis de $20 \mathrm{~kg} \mathrm{ha}^{-1}$. El mayor BPN (0.21 kg ha-1) se obtuvo con la dosis de $20 \mathrm{~kg} \mathrm{ha}^{-1}$ para el fraccionamiento $40-60 \%$, que fue 1.16 veces mayor que el BPN de la dosis de $20 \mathrm{~kg} \mathrm{ha}^{-1}$ $\left(0.18 \mathrm{~kg} \mathrm{ha}^{-1}\right)$ para el fraccionamiento de $100 \%$. Para la fracción 40-60 \% la disminución del BPN fue más abrupta con el incremento de la dosis de fósforo (1.9 y 4.2 veces, respectivamente) que con el fraccionamiento del $100 \%$, el cual fue más gradual (1.2 y 2.25 veces, respectivamente).
Los valores de BPN deben ser de 1 o ligeramente menor, ya que esto representaría sostenibilidad del sistema productivo (Dobermann, 2005).

El P es esencial para el crecimiento y desarrollo de las plantas. La mayoría de los suelos son deficientes en las formas de fósforo asimilables, por lo que se requiere la aplicación de fertilizantes fosfatados para alcanzar altos niveles de productividad; sin embargo, la mayor parte del 
P aplicado es rápidamente fijado en el suelo en fracciones que están pobremente disponibles para las raíces de las plantas. La interacción del fósforo con diferentes nutrientes del suelo es de gran importancia en la absorción, traslocación y utilización de este elemento por el vegetal. La nutrición inadecuada de P podría provocar alteraciones fisiológicas en las plantas; éstas se consideran eficientes cuando en el aprovechamiento de este nutriente, aún bajo determinadas condiciones nutricionales, normales o adversas, consiguen utilizarlo para su crecimiento y desarrollo (Fernández, 2007).

Según Fixen et al. (2015), los niveles bajos sugieren que los cambios en el manejo podrían mejorar la eficiencia y la fertilidad del suelo, mientras que con niveles altos la fertilidad del suelo podría disminuir.

\section{CONCLUSIONES}

El comportamiento es inversamente proporcional entre la dosis y el fraccionamiento aplicado; así, la dosis y el fraccionamiento incrementan la eficiencia agronómica, el factor parcial de productividad y el balance parcial de nutrientes. La relación entre el rendimiento y la eficiencia agronómica de $\mathrm{P}$ es cuadrática, por lo que pueden generarse recomendaciones apropiadas para la fertilización de los cultivos. Este estudio contribuye al manejo de los nutrimentos para lograr mayor producción y eficiencia agronómica del agrosistema plátano.

\section{BIBLIOGRAFÍA}

Adamtey N., M. W. Musyoka, C. Zundel, J. G. Cobo, E. Karanja, K. K. M. Fiaboe, ... and D. Foster (2016) Productivity, profitability and partial nutrient balance in maize-based conventional and organic farming systems in Kenya. Agriculture, Ecosystems and Environment 235:61-79, https://doi.org/10.1016/j.agee.2016.10.001

Avellán L., Á. M. Calvache U. y N. Cabeña (2015) Curva de absorción de nutrientes por el cultivo de plátano barraganete (Musa paradisiaca L.). TsaFiqui Revista de Investigación Científica 7:15-29, https://doi.org/10.29019/tsafiqui.v0i7.271

Bhattacharya A. (2018) Changing Climate and Resource Use Efficiency in Plants. Academic Press. London, UK. pp:241-305.

Bertsch F. (2003) Absorción de Nutrimentos por los Cultivos. Asociación Costarricense de la Ciencia del Suelo. San José, Costa Rica. $307 \mathrm{p}$.

Boaretto A., T. Muraoka y P. Trevelin (2007) Uso eficiente del nitrógeno de los fertilizantes convencionales. Informaciones Agronômicas 120:13-14

Bruulsema T. W., C. Witt, F. García, S. Li, T. N. Rao, F. Chen and S. Ivanova (2008) A global framework for fertilizer BMPs. Better Crops with Plant Food 92:13-15.

Castillo G. A. M., J. A. Hernández M., E. Avitia G., J. Pineda P., L. A. Valdéz A. y T. Corona T. (2011) Extracción de macronutrientes en banano 'Dominico' (Musa spp.). Phyton 80:65-72

Ciampitti I. A. y F. 0. García (2008) Balance y eficiencia de uso de los nutrientes en sistemas agrícolas. Revista Horizonte A 4:22-28.

Combatt E. M., G. Martínez y J. L. Barrera (2004) Efecto de la interacción de $\mathrm{N}$ y K sobre las variables de rendimiento del cultivo de plátano (Musa AAB Simmonds) en San Juan de Urabá - Antioquia. Temas Agrarios 9:5-12, https://doi.org/10.21897/rta.v9i1.618

Cruzate G. A. y R. Casas (2003) Balance de nutrientes. Revista Fertilizar
INTA 8:7-13.

Dixon J., A. Gulliver and D. Gibbon (2001) Farming Systems and Poverty: Improving Farmers' Livelihoods in a Changing World. FAO and World Bank. Rome, Italy and Washington D. C., USA. 412 p.

Dobermann A. R. (2005) Nitrogen use efficiency-state of the art Agronomy \& Horticulture Faculty Publication 316. University of Nebraska. Lincoln, Nebraska, USA. 16 p.

Doyle A. D. and I. C. R. Holford (1993) The uptake of nitrogen by wheat, its agronomic efficiency and their relationship to soil and fertilizer nitrogen. Australian Journal of Agricultural Research 44:12451258, https://doi.org/10.1071/AR9931245

Espinosa J. y J. P. García (2009) Herramientas para mejorar la eficiencia de uso de nutrientes en maíz. In: Memoria del Simposio Uso Eficiente de Nutrientes. J. Espinosa y J. P. García (eds.) Realizado del 16 al 20 de noviembre de 2009. International Plant Nutrition Institute. San José Costa Rica. pp:49-56.

Fageria N. K., V. C. Baligar, A. Moreira and T. A. Portes (2010) Dry bean genotypes evaluation for growth, yield components and phosphorus use efficiency. Journal of Plant Nutrition 33:21672181, https://doi.org/10.1080/01904167.2010.519089

Fallah S. and A. Tadayyon (2010) Uptake nitrogen efficiency in forage maize: effects of nitrogen and plant density. Agrociencia 44:549-560.

FAOSTAT, Food and Agriculture Organization of the United Nations Statistical Database (2018) Food and agriculture data. Food and Agriculture Organization of the United Nations. Rome. http://www.fao.org/ faostat/en/\#home (December 2018).

Fernández M. T. (2007) Fósforo: amigo o enemigo. ICIDCA. Sobre los Derivados de la Caña de Azúcar 41:51-57.

Fixen P. E. (2010) Eficiencia de uso de nutrientes en el contexto de agricultura sostenible. Informaciones Agronómicas 76:1-9.

Fixen P., F. Brentrup, T. Bruulsema, F. Garcia, R. Norton and S. Zingore (2015) Nutrient/fertilizer use efficiency: measurement, current situation and trends. In: Managing Water and Fertilizer for Sustainable Agricultural Intensification. P. Drechsel, P. Heffer, H. Magen, R. Mikkelsen and D. Wichelns (eds.). International Fertilizer Industry Association (IFA), International Water Management Institute (IWMI), International Plant Nutrition Institute (IPNI) and International Potash Institute (IPI). Paris, France. pp:8-38.

Folberth C., H. Yang, T. Gaiser, J. Liu, X. Wang, J. Williams and R. Schulin (2014) Effects of ecological and conventional agricultural intensification practices on maize yields in sub-Saharan Africa under potential climate change. Environmental Research Letters 9:044004, https://doi.org/10.1088/1748-9326/9/4/044004

Furcal-Beriguete P. y A. Barquero-Badilla (2013) Respuesta del plátano a la fertilización con P, K y S durante el primer ciclo productivo. Agronomía Mesoamericana 24:317-327.

Gourley C. J. P., D. L. Allan and M. P. Russelle (1994) Plant nutrient efficiency: a comparison of definitions and suggested improvement. Plant and Soil 158:29-37, https://doi.org/10.1007/BF00007914

INAMHI, Instituto Nacional de Meteorología e Hidrología (2017) Anuario Metereológico. No. 53-2013. Instituto Nacional de Meteorología e Hidrología. Quito, Ecuador. 150 p.

INEC, Instituto Nacional de Estadística y Censos (2014) Compendio Estadístico 2014. Instituto Nacional de Estadística y Censos. Quito, Ecuador. 289 p.

INEC, Instituto Nacional de Estadísticas y Censos (2018) Estadísticas agropecuarias. Encuesta de Superficie y Producción Agropecuaria Continua. Instituto Nacional de Estadísticas y Censos. Quito, Ecuador. http://www.ecuadorencifras.gob.ec/ institucional/home/ (Marzo 2019).

Lipiec J. and W. Stępniewski (1995) Effects of soil compaction and tillage systems on uptake and losses of nutrients. Soil and Tillage Research 35:37-52, https://doi.org/10.1016/0167-1987(95)00474-7

MAGAP, Ministerio de Agricultura, Ganaderia, Acuacultura y Pesca (2014) Boletin situacional banano 2014. Coordinación General del Sistema de Información Nacional. Ministerio de Agricultura, Ganaderia, Acuacultura y Pesca. Quito, Ecuador. 6 p.

Oenema J., S. Burgers, K. Verloop, A. Hooijboer, L. Boumans and $\mathrm{H}$. ten Berge (2010) Multiscale effects of management environmental conditions, and land use on nitrate leaching in dairy farms. Journal of Environmental Quality 39:2016-2028, https://doi.org/10.2134/jeq2010.0035 
Sánchez T. J. D., G. A. Ligarreto M. y F. R. Leiva B. (2012) Variabilidad del crecimiento y rendimiento del cultivo de maíz para choclo (Zea mays L.) como respuesta a diferencias en las propiedades químicas del suelo en la sabana de Bogotá, Colombia. Revista Facultad Nacional de Agronomía Medellín 65:6579-6583.

Sepúlveda W. S., I. Ureta, G. A. Hernández y G. K. Solorzano (2017) Consumo de plátano en Ecuador: hábitos de compra y disponibilidad a pagar de los consumidores. Revista em Agronegócio e Meio Ambiente, Maringá 10:995-1014, https://doi.org/10.17765/2176-9168.2017v10n4p995-1014

Stewart W. M. (2007) Consideraciones en el uso eficiente de nutrientes. Informaciones Agronómicas 67:1-6. van Leeuwen M. M. W. J., C. E. van Middelaar, J. Oenema, J. C. van Dam J. J. Stoorvogel, C. R. Stoof and I. J. M. de Boer (2019) The relevance of spatial scales in nutrient balances on dairy farms. Agriculture, Ecosystems and Environment 269:125139, https://doi.org/10.1016/j.agee.2018.09.026

Vivas-Cedeño J. S., J. O. Robles-García, I. González-Ramírez, D. A. Álava-Cruz y M. A. Meza-Loor (2018) Fertilización del plátano con nitrógeno, fósforo y potasio en cultivo establecido. Dominio de las Ciencias 4:633-647.

Weih M., L. Asplund and G. Bergkvist (2010) Conceptual framework for integrating aspects of nitrogen use efficiency in cereals. Aspects of Applied Biology 105:195-200. 